\title{
Bioinformatic Analysis of Prognostic Value of Pyroptosis-Related Genes and Its Effect on Immune Cell Infiltration in Pancreatic Adenocarcinoma
}

\author{
Jian Wang*, Hui Zhang (D**, Peng Shao, Xu Zhang, Bin Zhou \\ University, Nanjing, Jiangsu Province, People's Republic of China \\ *These authors contributed equally to this work \\ Correspondence: Xu Zhang; Bin Zhou, Tel/Fax +86-25-83284735, Email zhanmusil99I@I63.com; zbjszl@I26.com
}

Department of General Surgery, Jiangsu Cancer Hospital \& Jiangsu Institute of Cancer Research, The Affiliated Cancer Hospital of Nanjing Medical

\begin{abstract}
Purpose: Pancreatic adenocarcinoma has a poor prognosis and chemotherapy has its limitation due to tumor heterogeneity. It is essential to find novel targets involving in tumorigenesis of pancreatic adenocarcinoma. Growing evidences indicated that pyroptosis is involved in tumorigenesis of many cancers, but the relationship between pyroptosis and pancreatic adenocarcinoma still remains to be elucidated. Our object is to explore whether pyroptosis-related different expression genes have association with survivals of pancreatic adenocarcinoma patients and the mechanism they may participate in. Besides, we also analyzed their effect on immune cell infiltration in tumor microenvironment.

Patients and Methods: We used the bioinformatic analysis tool including GEPIA, cBioPortal, STRING, GeneMANIA, R software 4.03 and TIMER2.0 to investigate the different expression, prognostic value, protein-protein interaction, gene ontology, pathway and effect of immune cell infiltration of pyroptosis-related genes in PAAD.

Results: Many pyroptosis-related genes express differently between pancreatic adenocarcinoma and normal tissues and they are associated with survival of PAAD. GABARAP and IL18 may play a key role in tumorigenesis of PAAD, for they are connected with overall survival, disease free survival and pathological stages at the same time. The function of pyroptosis-related genes includes cytokine production, endopeptidase activity, regulation of inflammation and inflammasome complex and pyroptosis-related genes have effect on immune cells infiltration in PAAD microenvironment.

Conclusion: Lots of pyroptosis-related DEGs may get involved in pathogenesis of PAAD and their high expression have an effect on survival. GABARAP and IL18 could be valuable research targets of PAAD.
\end{abstract}

Keywords: pyroptosis, pyroptosis-related gene, pancreatic adenocarcinoma, immune cell, microenvironment

\section{Introduction}

Pancreatic adenocarcinoma (PAAD) is a high malignant cancer which has an awful prognosis. Among all malignancy, it ranks the tenth in terms of incidence but fourth in terms of cancer -related death and 5-year overall survival only reach $6 \%$. ${ }^{1,2}$ PAAD is a silent disease at early-stage and the appearance of jaundice often accompany the diagnosis of locally advanced unresectable tumors.Less than $20 \%$ patients meet the criteria for surgical resection. ${ }^{3}$ In addition, outcome of surgery is not satisfied and even early-stage PAAD can have metastasis, so it is regarded as a systemic disease which needs a comprehensive treatment. ${ }^{4}$ But due to tumor heterogeneity and robust drug resistance, chemotherapy also fails to bring a favorable outcome. So it is critical to identify new biologic factors which participate in tumorigenesis of PAAD. They could be the potential new markers which may be worth performing a further step research.

Pyroptosis is identified as a distinct programmed cell death from apoptosis, which could promote inflammation. The characteristics of pyroptosis include cell swelling, membrane rupture and proinflammatory factors releasing. Pyroptosis initiates when certain molecular patterns activate inflammasomes including NLR family, AIM2, Pyrin etc. 
Inflammasomes can cleave Caspase1 into the viable form, the latter can dictate GasderminD to form a pore in cell membrane and release IL18 and IL1 $\beta$ as the mature form. ${ }^{5,6}$ In addition to this canonical pathway, other Caspase family members also can induce pyroptosis like Caspase4, Caspase 5 and Caspase $8 .{ }^{7,8}$ Caspase 3 also can execute cell pyroptosis by activating GasderminE. ${ }^{9}$ Recently, novel evidences indicate that pyroptosis is involved in pathogenesis of cancer such as breast cancer, lung cancer, colon cancer etc. ${ }^{10-12}$ Activation of pyroptosis can suppress tumor growth, but on the other hand some studies find it can enhance tumorigenesis. ${ }^{13}$ It can also affect tumor immunity by regulating the immune cells level in tumor environment. ${ }^{14}$ However, whether pyroptosis is associated with the mechanism and prognosis of PAAD remains unclear. Thus, we performed a comprehensive analysis with the determination to uncover the prognostic value of pyroptosis-related genes and their effects on immune cell infiltration in patients with PAAD.

\section{Materials and Methods}

\section{Gene Expression Profiling Interactive Analysis (GEPIA)}

GEPIA (http://gepia.cancer-pku.cn/) is an interactive web server for analyzing the RNA sequencing expression based on data from the TCGA and the GTEx projects. ${ }^{15}$ In this study, we used the tool "Differential Expression Analysis" to perform a differential expression gene (DEGs) comparison between PAAD tumor and normal tissues. The analysis method selected ANOVA and $\log |\mathrm{FC}|$ Cutoff $>1$, q-value Cutoff $<0.05$ were set as the significance thresholds. The pyroptosis-related genes were first selected in previous literature as routine, ${ }^{16}$ then we overlapped the extracted pyroptosis-related genes with the DEGs. Next step, we analyzed the correlation between survival and pyroptosis-related genes by using Survival Plots tool with detailed setting to be Cut off-High 60\%, Cut off-low $40 \%$ and $95 \%$ Confidence Interval.

\section{cBioPortal}

cBioPortal (www.cbioportal.org) is an open website which integrates many data resource including The Cancer Genome Atlas (TCGA), The Cancer Genome Atlas (ICGC), Cancer cell line encyclopedia (CCLE) and American association cancer research (AACR) databases. The cBioPortal can perform a comprehensive analysis of genes or proteins for it consists of cancer-omic data including mutation, fusions, copy number variation. So we obtained the alteration informations of pyroptosis-related genes from cBioPortal.

\section{STRING}

STRING (https://string-db.org/) website focuses on protein functions and interactions, whose data includes 14,094 organisms, 67.6 millions proteins and 20 billions interactions and its goal is to achieve a comprehensive analysis and build an objective protein interaction network. ${ }^{17}$ We applied STRING to analyzed the protein-protein interactions between targeted proteins corresponding to the identified pyroptosis-related genes.

\section{GeneMANIA}

GeneMANIA (http://www.genemania.org) is a database focusing on genes function and related genes including proteinprotein, protein-DNA and genetic interactions, pathways, reactions, gene and protein expression data, protein domains and phenotypic screening profiles. We used GeneMANIA to search the prognostic pyroptosis-related DEGs genetic functions and interactions.

\section{R Software 4.03}

Based on these DEGs who are associated with prognosis of PAAD, gene ontology (GO) and Kyoto Encyclopedia of Genes and Genomes (KEGG) analysis were performed by applying the R package "clusterProfiler", "org.Hs.eg.db", "enrichplot" and "ggplot2". 


\section{Timer2.0}

TIMER (http://timer.cistrome.org/) is a comprehensive resource including four modules for investigating the associations between infiltration of immune cells and genes in multiple cancer types. ${ }^{18}$ In "Immune Association Module", we investigated the association between different expression of DEGs and infiltration levels of immune cells in PAAD.

\section{Results}

\section{Identification of Differentially Expressed Pyroptosis-Related Genes in PAAD Patients}

The 131 pyroptosis-related genes were selected from previous literatures (Supplementary Table 1). The differentially expressed genes in PAAD were analyzed on GEPIA website. There are 81 genes when we overlapped the pyroptosisrelated genes with DEGs (Supplementary Table 1). Among obtained genes, there were many significant pyroptosisrelated DEGs overexpressing in tumor compared with normal tissue, which may play an important role in mechanism and prognosis of PAAD. For instance, CASP4, IL18, HMGB1, GABARAP, MELK, NLRP1, PKM and TFEB (Figure 1).

\section{The Prognostic Value of Pyroptosis-Related DEGs in PAAD Cohort}

In next step, we performed correlation analysis between DEGs and PAAD survival in GEPIA. 15 pyroptosis-related DEGs were screened out to be associated with overall survival (Supplementary Table 2). Among these genes, the different expression of CASP4, IL18, HMGB1, GABARAP, MELK, NLRP1, PKM and TFEB had effects on both overall survival and disease free survival (Figure 2). What' more, IL18 and GABARAP expressed distinctly in different pathological stages (Supplementary Figure 1). Meanwhile, GDF11, ITPR1, PLCG1, CASP3, GSDMB, NEK7 and APAF1 were only related with overall survival (Supplementary Figure 2). From another perspective, high expression
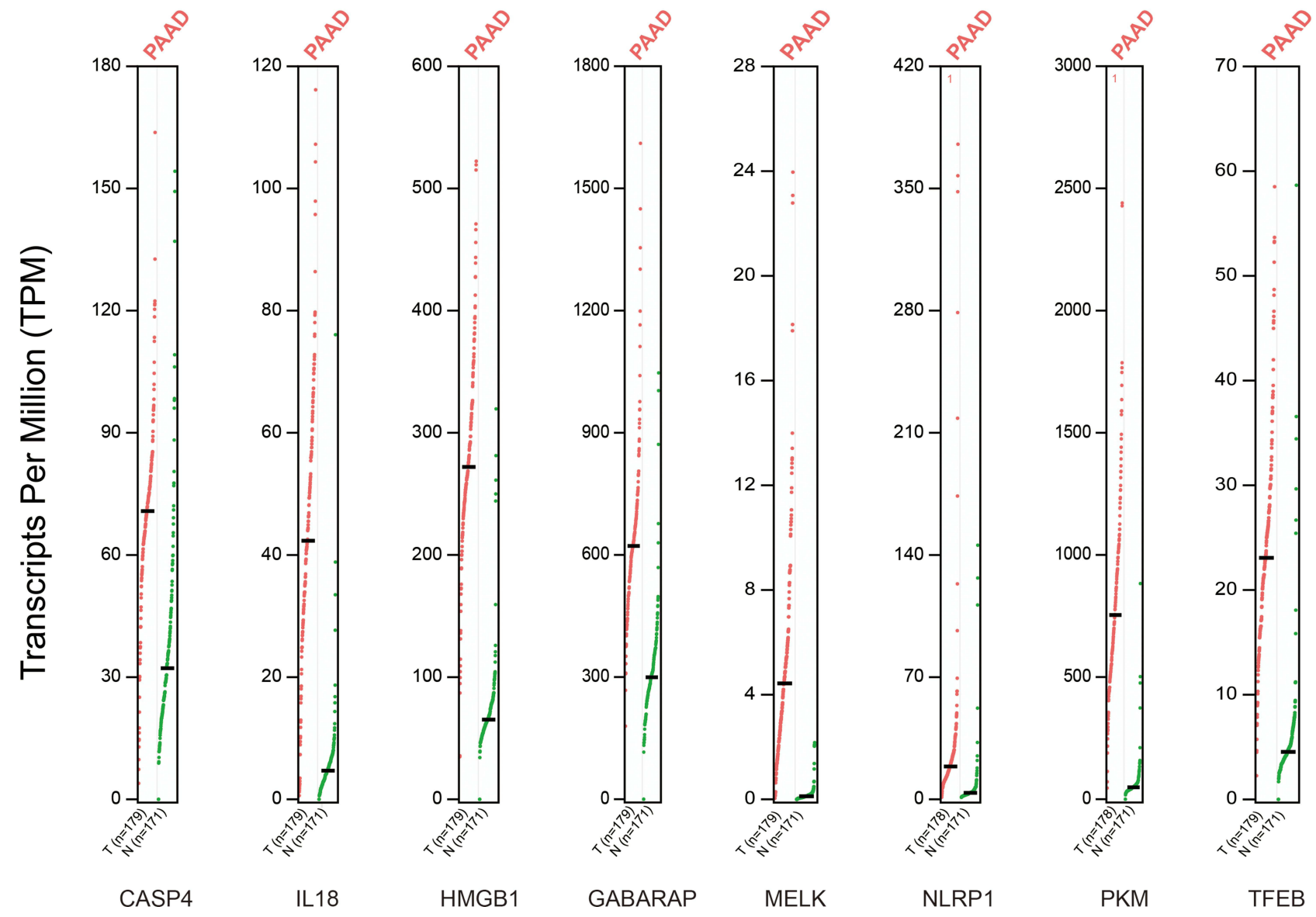

Figure I The expression profile of some selected pyroptosis related genes in PAAD. CASP4, ILI8, HMGBI, GABARAP, MELK, NLRPI, PKM and TFEB, the expression level of these pyroptosis related DEGs are higher in tumor than normal tissue. 

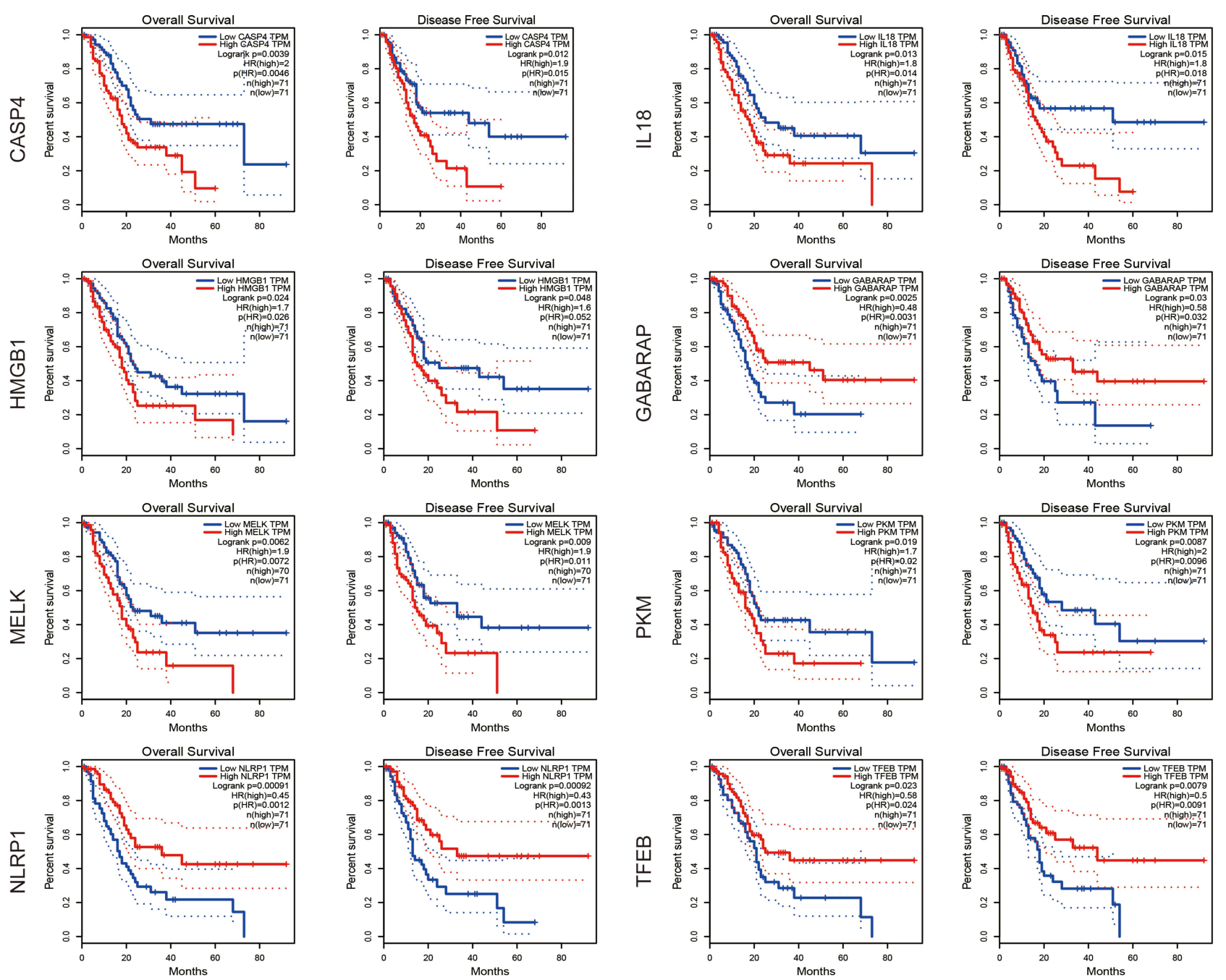

Figure 2 CASP4, ILI8, HMGBI, GABARAP, MELK, NLRPI, PKM and TFEB have prognostic value in PAAD. Higher expression of CASP4, ILI8, HMGBI, MELK and PKM are associated with short OS and DFS. Higher expression of GABARAP, NLRPI and TFEB make a contribution to a better OS and DFS.

of CASP4, IL18, HMGB1, MELK, PKM, CASP3, GSDMB and NEK7 had the negative correlations with OS. On the contrary, GABARAP, NLRP1, TFEB, GDF11, ITPR1, PLCG1 and APAF1 could improve prognosis of PAAD by overexpressing. The analysis results demonstrated that pyroptosis-related DEGs are associated with the prognosis of PAAD and activation of different pyroptosis pathway may lead to converse outcome of survival.

\section{The Function of Pyroptosis-Related DEGs in PAAD Pathogenesis}

We detected the alterations of pyroptosis-related DEGs in PAAD by using cBioPortal website. The prognosis-related genes, CASP4, IL18, HMGB1, GABARAP, MELK, NLRP1, PKM and TFEB altered in $0.6 \%, 0.6 \%, 0.4 \%, 1.4 \%, 1.8 \%$, $1.5 \%, 1 \%$ and $1.7 \%$ in PAAD patients. Totally, 92 samples had alterations recorded in 850 samples. Amplification and deep deletion accounted for major proportion in alterations (Figure 3A and B). The function of genes and interactions between corresponding proteins are important elements for understanding the biological phenomenon of cell, then we conducted PPI, GO and KEGG pathway analysis of these pyroptosis-related DEGs. From results we can see that IL18 has direct interactions with many proteins in pyroptosis pathway. GABARAP was isolated from the main network and seems to be a regulatory factor rather than direct engagement. Based on GeneMANIA and STRING data, functions of the prognostic pyroptosis related DEGs include cytokine production, endopeptidase activity, regulation of inflammation, inflammasome complex and tumor necrosis et al (Figure 3C, D and Supplementary Figure 3). The function of the 

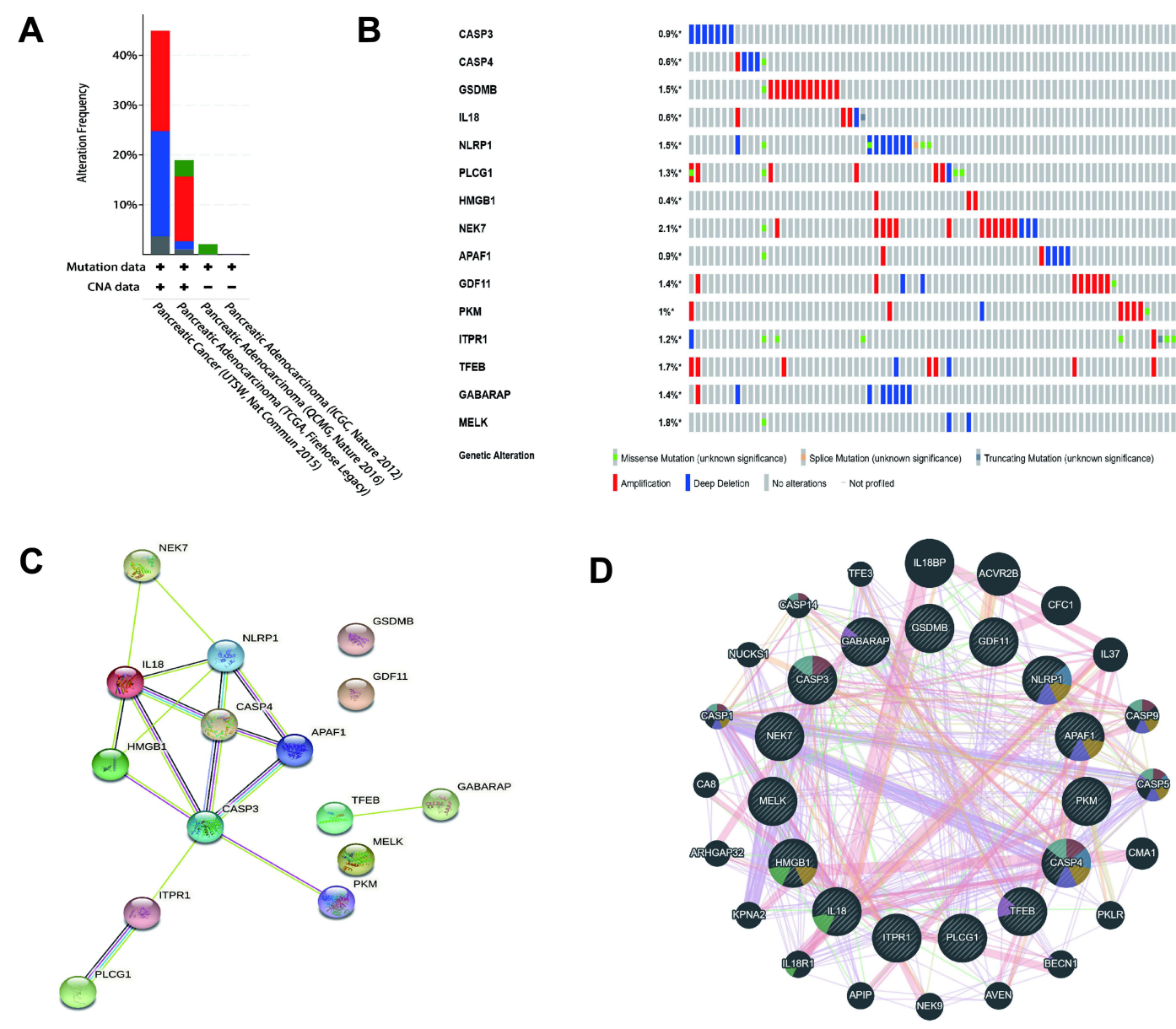

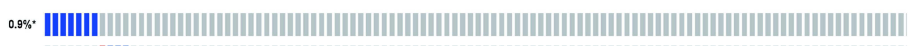
. $0.6 \%$.

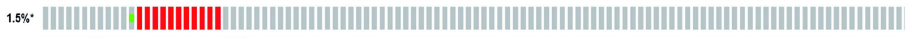
0.6\%

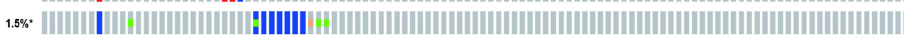
1.3\%. A .4.8\%

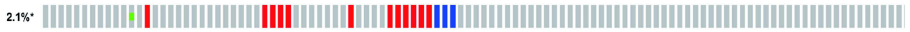

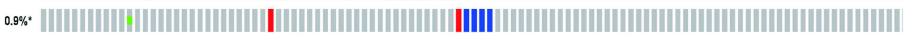

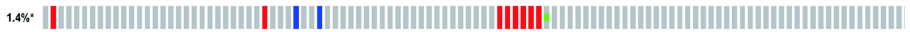

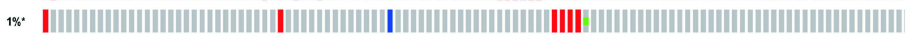

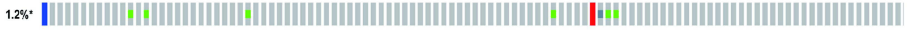

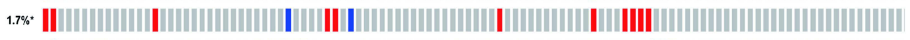

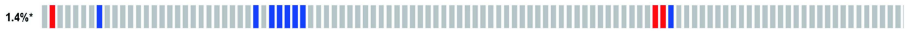

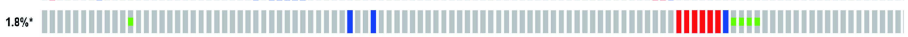

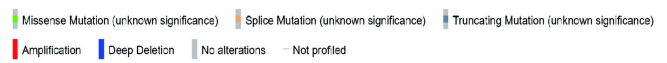

Figure 3 The mutation and function of pyroptosis related DEGs. (A and $\mathbf{B}$ ) The summary of pyroptosis related DEGs mutation in PAAD. (C and D) The functions and protein-protein interactions of pyroptosis related DEGs in PAAD.

prognostic pyroptosis related DEGs indicated that it may play a significant role in regulating inflammation and immune in PAAD.

\section{Pyroptosis-Related Genes Effect on Immune Cell Infiltration in PAAD}

We further explored the effect of pyroptosis-related genes on immune cells infiltration in PAAD by applying TIMER2.0. The results indicated that prognostic pyroptosis-related DEGs are closely connected with infiltrations of immune cells (Figure 4, Supplementary Figure 4) except that there are no data resource about MELK, PKM and TFEB in TIMER2.0 website. For instance, CASP4 expression was associated with $\mathrm{CD}^{+} \mathrm{T}$ cell, $\mathrm{CD} 4^{+} \mathrm{T}$ cell, B cell, DC cell and neutrophil cell level. HGMB1 had an effect on infiltration levels of $\mathrm{CD} 8^{+} \mathrm{T}$ cell, $\mathrm{CD} 4^{+} \mathrm{T}$ cell, B cell, DC cell, neutrophil cell and macrophage cell. IL18 was only connected with infiltration levels of $\mathrm{CD} 8^{+} \mathrm{T}$ cell, B cell and neutrophil cell in PAAD. GABARAP had connections with the infiltrations of $\mathrm{CD} 8^{+} \mathrm{T}$ cell, DC cell, macrophage cell and neutrophil cell. NLRP1 affected the infiltration levels of $\mathrm{CD} 8^{+} \mathrm{T}$ cell, $\mathrm{CD} 4^{+} \mathrm{T}$ cell, $\mathrm{B}$ cell, DC cell, neutrophil cell and macrophage cell and the other OS related genes all have connections with infiltrating levels of certain immune cells. The pyroptosis-related DEGs may influence tumor microenvironment by regulating immune cells infiltration.

\section{Discussion}

For a more in-depth exploration of cell death in cancer, pyroptosis has become a hot topic in cancer research due to its function in tumorigenesis and potential target value in therapy strategy. Up to now, there many researches revealed that cancer cells can die of pyroptosis such as esophageal carcinoma, breast cancer, ovarian cancer, lung cancer and colon 

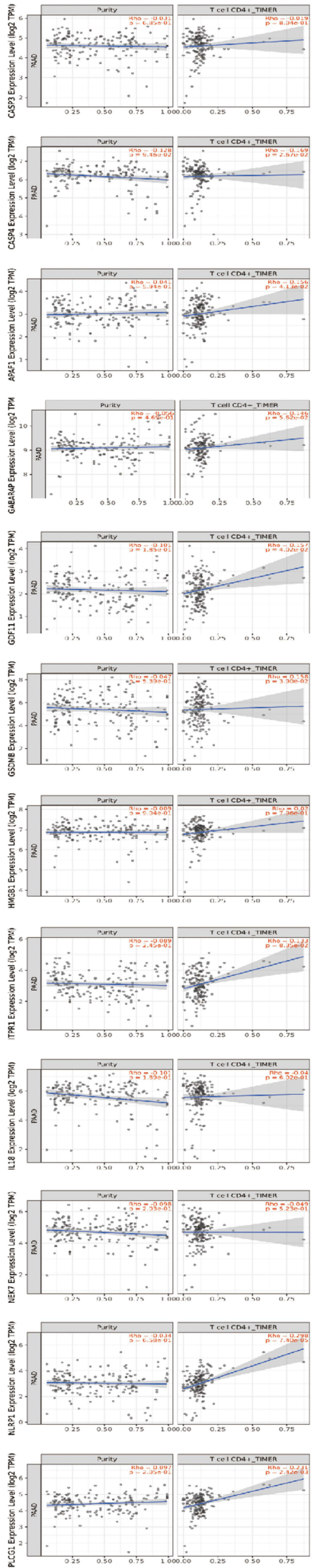
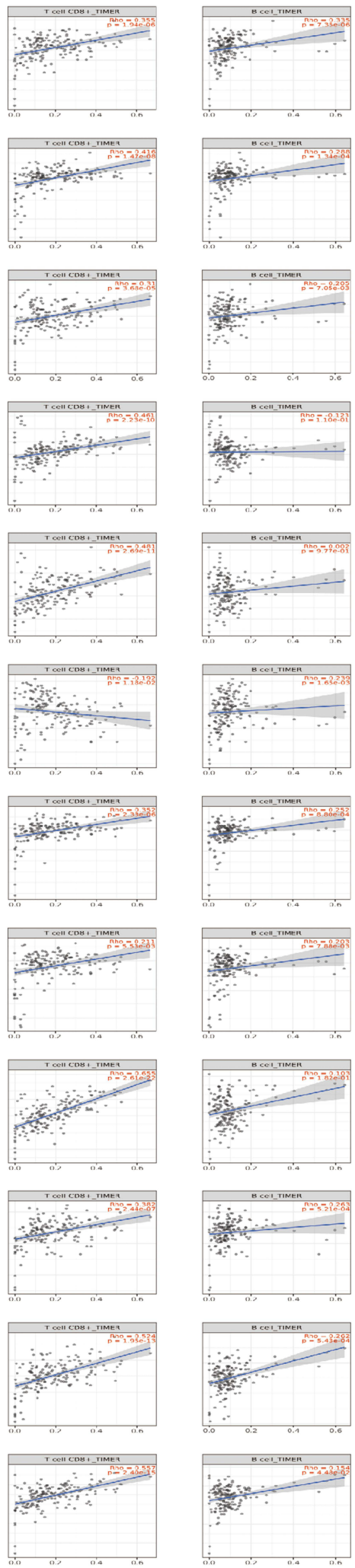

Immune cells infiltration leve
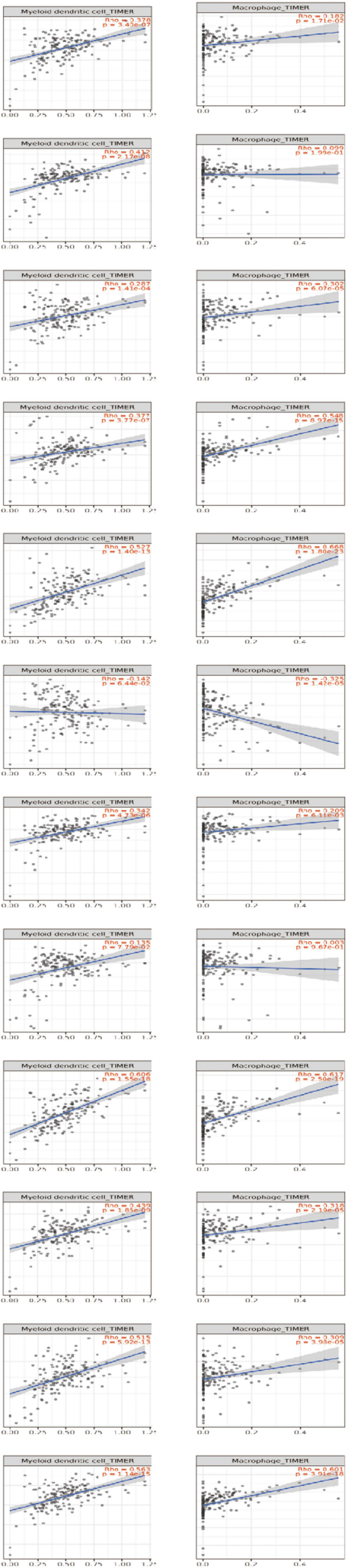
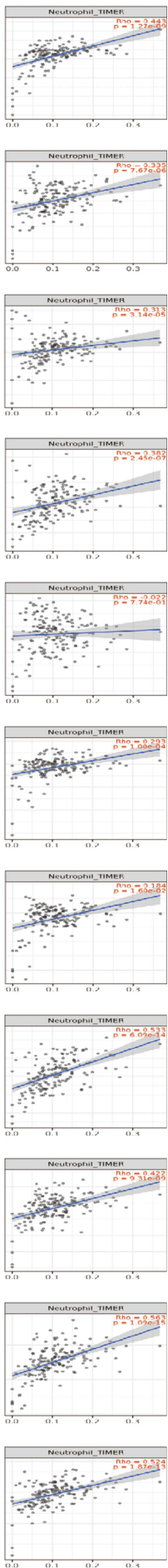

Figure 4 The effect of pyroptosis related DEGs on immune cells infiltration level. 
cancer $^{10,19-21}$ and studies also indicated that chemotherapy drugs and other reagents can trigger pyroptosis in various cancer. ${ }^{22}$ But whether pyroptosis is involved in mechanism of PAAD remains to be elucidated. So we did a comprehensive analysis of pyroptosis-related DEGs in PAAD from OS, DFS, pathological stage, gene alteration, GO, KEGG, PPI and immune cell infiltration aspects. In our results, CASP4, HMGB1, MELK, NLRP1, PKM, TFEB, IL18 and GABARAP are associated with OS and DFS. Besides, GDF11, ITPR1, PLCG1, CASP3, GSDMB, NEK7, APAF1 only have the connections with OS. In addition to OS and DFS, GABARAP and IL18 express differently according to pathological stage at the same time, suggesting they may play a key role in tumorigenesis of PAAD.

As known, GABARAP is an important protein family which serves as a core character in autophagy. ${ }^{23}$ But GABARAP might also get involved in mechanism of pyroptosis. It has been demonstrated that GABARAP plays a critical role in down-regulation of macrophage pyroptosis by suppressing GBP2-dependent Caspase11-induced inflammation in sepsis. ${ }^{24}$ The deficient of GABARAP also can enhance NLRP3 activation, Caspase1 activation and IL18, IL1 $\beta$ releasing in LPS-treated macrophages. ${ }^{25}$ Based on the above evidences, the high expression of GABARAP may suppress pyroptosis.

In previous literature, it has been indicated that GABARAP has prognostic value in different type tumors. High GABARAP expression is associated with poor prognosis and with pathological characteristics in breast cancer. ${ }^{26}$ But another study result suggested that GABARAP can suppress progression of breast cancer. ${ }^{27,28}$ GABARAP expresses differently between Glioma and normal brain tissue and has a prognostic value in Glioma patient. ${ }^{29}$ GABARAP has a relation with tumorigenesis of melanoma and ablation of GABARAP in mice suppresses tumor growth. ${ }^{30}$ GABARAP expresses lower in colorectal cancer compared to adjacent mucosa and may delay progression of colorectal cancer. ${ }^{31}$ Hitherto, no research has examined the mechanism and prognostic significance of GABARAP in PAAD, our analysis suggested that high level of expression of this gene is connected to good survival in PAAD patients and the potential mechanism is that GABARAP may serve as a pyroptosis suppressor in PAAD.

IL18 is a released cytokine at the terminal downstream of pyroptosis. In company with the pyroptosis iconic genes like CASP3, CASP4, GSDMB, high expression of IL18 may present that there is an enormous amount of pyroptosis in PAAD. The significant role of IL18 is inducing INF- $\gamma$ production and mediating Th1, Th2 response. ${ }^{32}$ The function of IL18 in mediating immune system makes it to be a double edge sword in tumorigenesis. IL18 has anti-tumor effect and it also can promote tumorigenesis by influencing angiogenesis, metastasis. ${ }^{33}$ There have been many studies investigating the correlation of IL18 with pancreatic carcinoma prognosis. Guo et al indicated that higher IL18 in pancreatic carcinoma tissues is associated with shorter overall survival while elevated serum level of IL18 is connected to longer survival. ${ }^{34}$ But another study demonstrated that elevated serum level of IL18 is connected to poor survival of pancreatic carcinoma patients. ${ }^{35}$ The role of IL18 in pancreatic carcinoma seems to be controversial and needs to be further explored. IL-18BP is a high-affinity IL-18 decoy receptor which is highly expressed in diverse tumors and the combination of IL-18 and IL18BP decreases activity of IL-18. ${ }^{36}$ Carbone et al study suggested that despite elevated concentration of IL-18BP and IL-18, elevation of free IL-18 in serum of pancreatic carcinoma patients is associated with poor survival. ${ }^{37}$ In our analysis results, high expression of IL18 makes contribution to shorter survival of PAAD patients. As mentioned above, we hypothesize that IL18 releasing by activation of pyroptosis is far more than IL-18BP and the free IL18 make progression of PAAD.

The different kind cells and extracellular matrix surrounding cancer cells are defined as tumor microenvironment. Tumor microenvironment is a revolutionary concept in cognition of tumorigenesis of cancer for realizing the important interaction between tumor cells and the microenvironment around them. So changing the status of tumor microenvironment can influence therapeutic efficacy significantly. ${ }^{38}$ Pyroptosis could releases various inflammatory cytokines maintaining inflammation and it has shown to be a two-side sword in tumor microenvironment dependent on the context, timing, and response degree. ${ }^{39}$ Our results showed that high expression of pyroptosis related DEGs have an effect on immune cells infiltration which indicated that pyroptosis related DEGs are associated with tumor microenvironment in PAAD. Meanwhile, immunotherapy treatment efficacy is limited in pancreatic cancer because of the functional and structural barrier imposed by the stroma components. ${ }^{40}$ So bodies of cell death induced by pyroptosis may contribute to the structural barrier in the tumor microenvironment. 


\section{Conclusion}

Pyroptosis-related DEGs have an obvious prognostic value in PAAD and have an effect on immune cell infiltration in tumor microenvironment. Especially, IL18 and GABARAP could be the most valuable genes of these pyroptosis-related DEGs, for they have association with OS, DFS and pathological stage at the same time. GABARAP and IL18 could be new valuable targets of PAAD research in next step. The limitation of our study is that we only carry out bioinformatics analysis but without further exploration of mechanism of pyroptosis-related DEGs in PAAD for lacking of laboratory supporting.

\section{Abbreviations}

PAAD, Pancreatic adenocarcinoma; DEGs, differential expression gene;

GEPIA, Gene Expression Profiling Interactive Analysis; TCGA, The Cancer Genome Atlas; ICGC, The Cancer Genome Atlas; GO, gene ontology; KEGG, Kyoto Encyclopedia of Genes and Genomes.

\section{Ethics Exemption}

The study is a secondary analysis research. The method and materials are all from public data source. The study is exempt from ethics approval. It has been confirmed by the Ethics Committee and Science and Technology Department of Jiangsu cancer hospital.

\section{Acknowledgments}

Jian Wang and Hui Zhang are co-first authors for this study.

\section{Disclosure}

The authors report no conflicts of interest in this work.

\section{References}

1. Ryan DP, Hong TS, Bardeesy N. Pancreatic adenocarcinoma. N Engl J Med. 2014;371(11):1039-1049. doi:10.1056/NEJMra1404198

2. Siegel R, Naishadham D, Jemal A. Cancer statistics, 2013. CA Cancer J Clin. 2013;63(1):11-30. doi:10.3322/caac.21166

3. Konstantinidis IT, Warshaw AL, Allen JN, et al. Pancreatic ductal adenocarcinoma: is there a survival difference for R1 resections versus locally advanced unresectable tumors? What is a "true" R0 resection? Ann Surg. 2013;257(4):731-736. doi:10.1097/SLA.0b013e318263da2f

4. Sohal DPS, Walsh RM, Ramanathan RK, Khorana AA. Pancreatic adenocarcinoma: treating a systemic disease with systemic therapy. $J$ Natl Cancer Inst. 2014;106(3):dju011. doi:10.1093/jnci/dju011

5. He W-T, Wan H, Hu L, et al. Gasdermin D is an executor of pyroptosis and required for interleukin-1 $\beta$ secretion. Cell Res. 2015;25(12):1285-1298. doi:10.1038/cr.2015.139

6. Broz P, Pelegrín P, Shao F. The gasdermins, a protein family executing cell death and inflammation. Nat Rev Immunol. 2020;20(3):143-157. doi:10.1038/s41577-019-0228-2

7. Gong W, Shi Y, Ren J. Research progresses of molecular mechanism of pyroptosis and its related diseases. Immunobiology. 2020;225(2):151884. doi:10.1016/j.imbio.2019.11.019

8. Sarhan J, Liu BC, Muendlein HI, et al. Caspase-8 induces cleavage of gasdermin D to elicit pyroptosis during infection. Proc Natl Acad Sci U S A. 2018;115(46):E10888-E10897. doi:10.1073/pnas.1809548115

9. Wang Y, Gao W, Shi X, et al. Chemotherapy drugs induce pyroptosis through caspase-3 cleavage of a gasdermin. Nature. 2017;547(7661):99-103. doi:10.1038/nature22393

10. Pizato N, Luzete BC, Kiffer LF, et al. Omega-3 docosahexaenoic acid induces pyroptosis cell death in triple-negative breast cancer cells. Sci Rep. 2018;8(1):1952. doi:10.1038/s41598-018-20422-0

11. Chen L, Weng B, Li H, et al. A thiopyran derivative with low murine toxicity with therapeutic potential on lung cancer acting through a NF- $\mathrm{KB}$ mediated apoptosis-to-pyroptosis switch. Apoptosis. 2019;24(1-2):74-82. doi:10.1007/s10495-018-1499-y

12. Yu J, Li S, Qi J, et al. Cleavage of GSDME by caspase-3 determines lobaplatin-induced pyroptosis in colon cancer cells. Cell Death Dis. 2019;10 (3):193. doi:10.1038/s41419-019-1441-4

13. Karki R, Kanneganti T-D. Diverging inflammasome signals in tumorigenesis and potential targeting. Nat Rev Cancer. 2019;19(4):197-214. doi:10.1038/s41568-019-0123-y

14. Minton K. Pyroptosis heats tumour immunity. Nat Rev Drug Discov. 2020;19(5):309. doi:10.1038/d41573-020-00062-8

15. Tang Z, Li C, Kang B, Gao G, Li C, Zhang Z. GEPIA: a web server for cancer and normal gene expression profiling and interactive analyses. Nucleic Acids Res. 2017;45(W1):W98-W102. doi:10.1093/nar/gkx247

16. Ye Y, Dai Q, Qi H. A novel defined pyroptosis-related gene signature for predicting the prognosis of ovarian cancer. Cell Death Discov. 2021;7 (1):71. doi:10.1038/s41420-021-00451-x

17. Szklarczyk D, Gable AL, Lyon D, et al. STRING v11: protein-protein association networks with increased coverage, supporting functional discovery in genome-wide experimental datasets. Nucleic Acids Res. 2019;47(D1):D607-D613. doi:10.1093/nar/gky1131 
18. Li T, Fu J, Zeng Z, et al. TIMER2.0 for analysis of tumor-infiltrating immune cells. Nucleic Acids Res. 2020;48(W1):W509-W514. doi:10.1093/ nar/gkaa407

19. Wang L, Li K, Lin X, et al. Metformin induces human esophageal carcinoma cell pyroptosis by targeting the miR-497/PELP1 axis. Cancer Lett. 2019;450:22-31. doi:10.1016/j.canlet.2019.02.014

20. Qiao L, Wu X, Zhang J, et al. $\alpha$-NETA induces pyroptosis of epithelial ovarian cancer cells through the GSDMD/caspase-4 pathway. FASEB J. 2019;33(11):12760-12767. doi:10.1096/fj.201900483RR

21. Zhang -C-C, Li C-G, Wang Y-F, et al. Chemotherapeutic paclitaxel and cisplatin differentially induce pyroptosis in A549 lung cancer cells via caspase-3/GSDME activation. Apoptosis. 2019;24(3-4):312-325. doi:10.1007/s10495-019-01515-1

22. Zheng Z, Li G. Mechanisms and therapeutic regulation of pyroptosis in inflammatory diseases and cancer. Int J Mol Sci. 2020;21(4):1456. doi:10.3390/ijms21041456

23. Kirkin V, Rogov VV. A diversity of selective autophagy receptors determines the specificity of the autophagy pathway. Mol Cell. 2019;76(2):268285. doi:10.1016/j.molcel.2019.09.005

24. Sakaguchi N, Sasai M, Bando H, et al. Role of Gate-16 and Gabarap in prevention of caspase-11-dependent excess inflammation and lethal endotoxic shock. Front Immunol. 2020;11:561948. doi:10.3389/fimmu.2020.561948

25. Zhang Z, Xu X, Ma J, et al. Gene deletion of Gabarap enhances Nlrp3 inflammasome-dependent inflammatory responses. J Immunol. 2013;190 (7):3517-3524. doi:10.4049/jimmunol.1202628

26. Bortnik S, Tessier-Cloutier B, Leung S, et al. Differential expression and prognostic relevance of autophagy-related markers ATG4B, GABARAP, and LC3B in breast cancer. Breast Cancer Res Treat. 2020;183(3):525-547. doi:10.1007/s10549-020-05795-Z

27. Liu Y, Wang D, Lei M, et al. GABARAP suppresses EMT and breast cancer progression via the AKT/mTOR signaling pathway. Aging. 2021;13 (4):5858-5874. doi:10.18632/aging.202510

28. Hervouet E, Claude-Taupin A, Gauthier T, et al. The autophagy GABARAPL1 gene is epigenetically regulated in breast cancer models. BMC Cancer. 2015;15:729. doi:10.1186/s12885-015-1761-4

29. Xu Y, Li R, Li X, et al. An autophagy-related gene signature associated with clinical prognosis and immune microenvironment in gliomas. Front Oncol. 2020;10:571189. doi:10.3389/fonc.2020.571189

30. Salah FS, Ebbinghaus M, Muley VY, et al. Tumor suppression in mice lacking GABARAP, an Atg8/LC3 family member implicated in autophagy, is associated with alterations in cytokine secretion and cell death. Cell Death Dis. 2016;7:e2205. doi:10.1038/cddis.2016.93

31. Gil J, Ramsey D, Pawlowski P, et al. The influence of tumor microenvironment on ATG4D gene expression in colorectal cancer patients. Med Oncol. 2018;35(12):159. doi:10.1007/s12032-018-1220-6

32. Kaplanski G. Interleukin-18: biological properties and role in disease pathogenesis. Immunol Rev. 2018;281(1):138-153. doi:10.1111/imr.12616

33. Bent R, Moll L, Grabbe S, Bros M. Interleukin-1 Beta-A friend or foe in malignancies? Int J Mol Sci. 2018;19(8):2155. doi:10.3390/ijms19082155

34. Guo X, Zheng L, Jiang J, et al. Blocking NF-kB is essential for the immunotherapeutic effect of recombinant IL18 in pancreatic cancer. Clin Cancer Res. 2016;22(23):5939-5950. doi:10.1158/1078-0432.CCR-15-1144

35. Bellone G, Smirne C, Mauri FA, et al. Cytokine expression profile in human pancreatic carcinoma cells and in surgical specimens: implications for survival. Cancer Immunol Immunother. 2006;55(6):684-698. doi:10.1007/s00262-005-0047-0

36. Zhou T, Damsky W, Weizman O, et al. IL-18BP is a secreted immune checkpoint and barrier to IL-18 immunotherapy. Nature. 2020;583 (7817):609-614. doi:10.1038/s41586-020-2422-6

37. Carbone A, Vizio B, Novarino A, et al. IL-18 paradox in pancreatic carcinoma: elevated serum levels of free IL-18 are correlated with poor survival. J Immunother. 2009;32(9):920-931. doi:10.1097/CJI.0b013e3181b29168

38. Wu T, Dai Y. Tumor microenvironment and therapeutic response. Cancer Lett. 2017;387:61-68. doi:10.1016/j.canlet.2016.01.043

39. Du T, Gao J, Li P, et al. Pyroptosis, metabolism, and tumor immune microenvironment. Clin Transl Med. 2021;11(8):e492. doi:10.1002/ctm2.492

40. Hester R, Mazur P, McAllister F. Immunotherapy in pancreatic adenocarcinoma: beyond "Copy/Paste". Clin Cancer Res. 2021;27:6287-6297. doi:10.1158/1078-0432.CCR-18-0900

International Journal of General Medicine

Dovepress

\section{Publish your work in this journal}

The International Journal of General Medicine is an international, peer-reviewed open-access journal that focuses on general and internal medicine, pathogenesis, epidemiology, diagnosis, monitoring and treatment protocols. The journal is characterized by the rapid reporting of reviews, original research and clinical studies across all disease areas. The manuscript management system is completely online and includes a very quick and fair peer-review system, which is all easy to use. Visit http://www.dovepress.com/testimonials.php to read real quotes from published authors.

Submit your manuscript here: https://www.dovepress.com/international-journal-of-general-medicine-journal 